Cien Saude Colet 2019; 24(1):345. ISSN 1413-8123

DOI: $10.1590 / 1413-81232018241.32242011$

\title{
TOTAL RETRACTION
}

The editorial team of Revista Ciência \& Saúde Coletiva communicates the formal publication of retraction for the extraction of the review article referred to below since it contains conceptual and quotation errors, which prejudices the trustworthiness of the information throughout the entire text.

Restrepo-Arango M; Gutierrez-Builes LA, Rios-Osorio LA. Food security in indigenous and peasant populations: a systematic review. Cien Saude Colet [online]. 2018; 23(4):1169-1181. Available from: $<$ http://www.scielo.br/scielo.php?script=sci_arttext\&pid=S1413-81232018000401169\&lng=pt\&nrm $=$ iso $>$. ISSN 1413-8123. http://dx.doi.org/10.1590/1413-81232018234.13882016. Available at: http:// www.scielo.br/scielo.php?script=sci_arttext\&pid=S1413-1232018000401169\&lng=pt\&nrm=iso

This text was translated into English: Restrepo-Arango M; Gutierrez-Builes LA, Rios-Osorio LA. Food security in indigenous and peasant populations: a systematic review. Cien Saude Colet [online]. 2018; 23(4):1169-1181. ISSN 1413-8123. http://dx.doi.org/10.1590/1413-81232018234.13882016.

Maria Cecília de Souza Minayo

Editor-in-chief 



\section{Food security in indigenous and peasant populations: a systematic review}

Marcos Restrepo-Arango ${ }^{1}$ Lina Andrea Gutiérrez-Builes ${ }^{1}$ Leonardo Alberto Ríos-Osorio ${ }^{2}$

${ }^{1}$ Grupo de Investigación Biología de Sistemas, Facultad de Medicina, Universidad Pontificia Bolivariana. Calle 78B Número 72a/109/Bloque B/cuarto piso. Medellín Colombia. marcos.restrepo@ upb.edu.co

${ }^{2}$ Grupo de investigación Salud y Sostenibilidad, Escuela de Microbiología, Universidad de Antioquia. Antioquia Colombia.

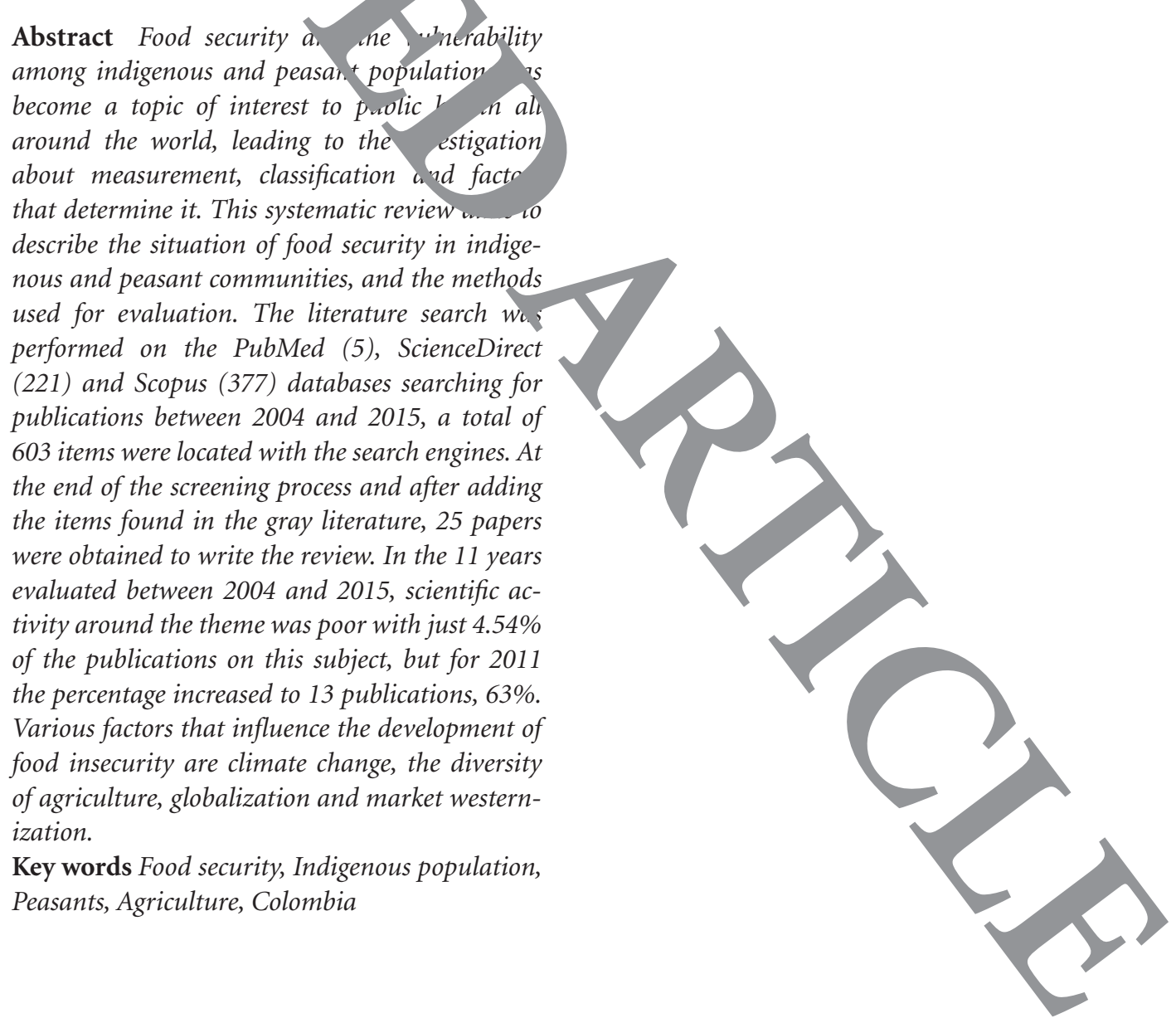




\section{Introduction}

The concept of food security has its origin in he mid-1970s, a period when the central focus ay und this concept was a government's capacity . . meet the energy and nutritional needs of a por ation through the adequate availability of $\mathrm{d}$ a d stability in the prices of basic prodcts at both national and international levels ${ }^{1}$. Subsequer his concept has been presented in divers ays by nternational organizations and resu che more than 200 different definitions ${ }^{-}$a these definitions have evolved since the com hencement the World Food Conference held th ticipating governments in 1974. During the 80s, this focus began to shift from the food cur ani ndividual nation and from increasing pply accor ing to demand to an approach that ought to in pr ve access to food by the end of tho decade ${ }^{2}$.

Subsequently, in 1990, these de ns were submitted to consensus at the Ad Foo A Summit, yielding the most recen concept pro sed by the Food and Agriculture Organizat: of the United Nations (FAO), which es ohe tha food security occurs when all peops have, at all times, physical and economic access to aificie safe, and nutritious food supplies to me eir needs and preferences and to lead an active ife as well as maintain a healthy diet ${ }^{3}$.

From a conceptual point of view, food security is supported by 4 pillars: food availability, access, use, and stability ${ }^{4}$. Availability refers to the physical supply of food and is determined by the levels of production, reserves, and trade; access refers to the economic and physical capacity to acquire food, which also depends on the market and not on availability alone; the dimension of use encompasses biological forms that provide most nutrients to the body; and stability connotes the balance of the other 3 determinants over time $e^{4}$.

Conversely, food insecurity has been defined as the limited or uncertain availability of nutritionally adequate and safe food or as the limited capacity to acquire food in socially acceptable ways $^{5}$. Consequently, food insecurity occurs when food systems are not accessible, not available, inadequate, or under stress ${ }^{6}$.

According to $\mathrm{FAO}$, indigenous and peasant populations are disproportionately affected by environmental degradation; economic and political marginalization; and activities promoting economic development that adversely affect the ecosystems, lifestyle, cultural heritage, and nutritional status of these populations ${ }^{4}$.
Therefore, the development of methods to evaluate the status of food security in indigenous and peasant populations is imperative along with research into whether and how the current political, economic, social, or environmental changes affect food security in these populations. In addition, the scope of parallel concepts, such as food sovereignty, which both involves and transcends issues related to food security, must be considered. In this regard, the Forum for the Food Sovereignty of 2007 has defined food sovereignty as the right of persons to healthy and culturally appropriate production through ecologically sustainable and rational methods and to define their own food and agricultural systems. Consequently, it can be inferred that food security represents a set of goals to be achieved, while food sovereignty implies an approach to achieve food security ${ }^{4}$.

Taking into account the diversity of existing theoretical and methodological perspectives in scientific literature for defining food security and the lack of consensus regarding the approach to study this concept in indigenous and peasant populations, we proposed a theoretical investigation with the objective of describing the status of food security and the methods used for its evalu. tion in these types of populations on the basis of systematic review of the scientific literature a period of 11 years.

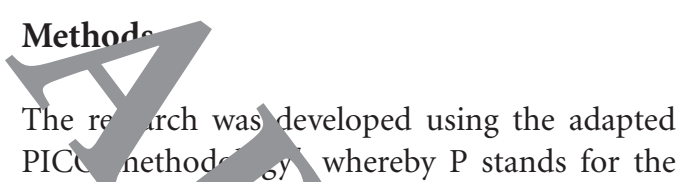
PIC nethod oy whereby P stands for the study popy $n$, in is case, the indigenous and peasant $\mathrm{ppu}$ th present vulnerability in their food curity; I re resents the methods used to evalua fe food s refers to existing food safety diagnoc and $\mathrm{O}$, cfers to the results of the review refled ing the dete nants of food security in these communit:

The problem ques defin $\varepsilon_{a r}$ the research process were as bllo What a the methods used for the evaluati 1 of food se urity in indigenous and peasant $p($ bulations; hoy is the diagnosis classified; and wh- detern ats have been described?

\section{Search strategy}

The study was structured in accordance ith the PRISMA statement ${ }^{8}$. A systematic literatu search was performed in the ScienceDirect, Scopus, and PubMed databases (January 2004-Jan- 
uary 2015). DeCS descriptors were employed o ensure sensitivity, descriptive non-DeCS descri tors were employed to ensure completeness, olean operators were combined to ensure ecific $y$.

The ch paths employed in each database are presente below. Grey literature was obtained througb Go gle Scholar search tool.

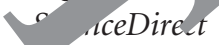

food secur" and ALL(" "standard methods" OR in ars R index) AND ("indigenous pop atio peasants OR native)).

Scopus

TITLE-Ab -KEY ("f ? security") AND ALL ("standard cm ds" $\mathrm{R}$ indicators OR INDEX) AND ALL ("in' cnous population" OR peasants OR native, ND $1 \mathrm{R}>2004$ AND (LIMIT-TO (DUC PE, “ar") AND ( LIMIT-TO (LANGUAGE "English"), AND ( LIMIT-TO (SRCTYPE, “j”)

PubMed

(("food security") AND ("standa sethod OR indicators OR index)) AND (in rgenous OR native)

Search results were imported to the 1 Reference Management Software, and du, icate were eliminated.

\section{Inclusion and exclusion criteria}

Only original articles published over a period of11years (2004-2015), written in English, containing information regarding indigenous and peasant populations, and containing descriptions or interpretations of the concept of food security from any measurement level, were included.

Articles that did not address the issue of food safety applied to the reference population of this study were excluded.

Data regarding each publication were extracted, and an information collection form, specifying the year, country, journal title, and the thematic issues of the journals, was created for the subsequent analysis.

\section{Results}

The review of scientific literature extracted from the 3 databases revealed 603 relevant articles published between January 2004 and January 2015 (ScienceDirect, 221; Scopus, 377; and PubMed, 5). Next, 19duplicate articles identified among the databases were eliminated using the Mendeley software. In total, 584 articles were evaluat- ed according to their titles and abstracts. From these, 553 articles were excluded because they did not meet the inclusion criteria described above. On analyzing the remaining 31 full-text articles, 9 were eliminated on the basis of exclusion criteria. Finally, 22 articles were obtained for analysis in the present systematic review (Figure 1).

Three articles from journals not indexed in the databases and identified using the Google Scholar were added to the 25 articles mentioned above, after meeting the inclusion and exclusion criteria established for the present review (Chart 1).

The scientific literature selected for this review comprised 25 articles published between January 2004 and January 2015, which were studies conducted in 8 different countries across 5 continents (Figure 2).

Food security has become a topic of common interest worldwide, and it has been approached in the most vulnerable communities, such as the inhabitants of rural areas. This is attributable to the severity of food insecurity in such communities and its impact on the health and economy of the countries. Despite this, the research identified sing our protocol was concentrated in countrie uch as the United Kingdom and the Unit-

stats, with $31.82 \%$ of the publications each, whereas ountries, such as Australia, Brazil, and South rica, yielded only $2.70 \%$ publications raphic 1).

These results demonstrate the importance that certain ton. present for researchers as well as the apa ity consistently address such topics and to ri ously al cate resources for their research. I this sen high proportion of studies regardin od se rity in the United States indicated hat ..... of he population in this country was d Ing with fo d insecurity in 2004 and 3.9\% with food ins at , and hunger during the same year ${ }^{9}$.

The journals with highest aber of publications on food insecurity y $\triangle \mathrm{MC}$ Public Health of the United Kin 1 , with $-63 \%$ of the publications (corresponding ${ }^{+}$articles, and The Journal of Nutrition, wit] $9.09 \%$. The main thematic focus of the 19 jou nals whose articles were included in the present medicine $(31.82 \%)$; followed by social scie (18.92\%); and finally, environmental, socia and biological sciences (16.22\%). This trend sugg that the issues regarding food insecurity that concern the scientific community the most include the impact of food insecurity on health as well as its social, biological, and environmental determinants. 

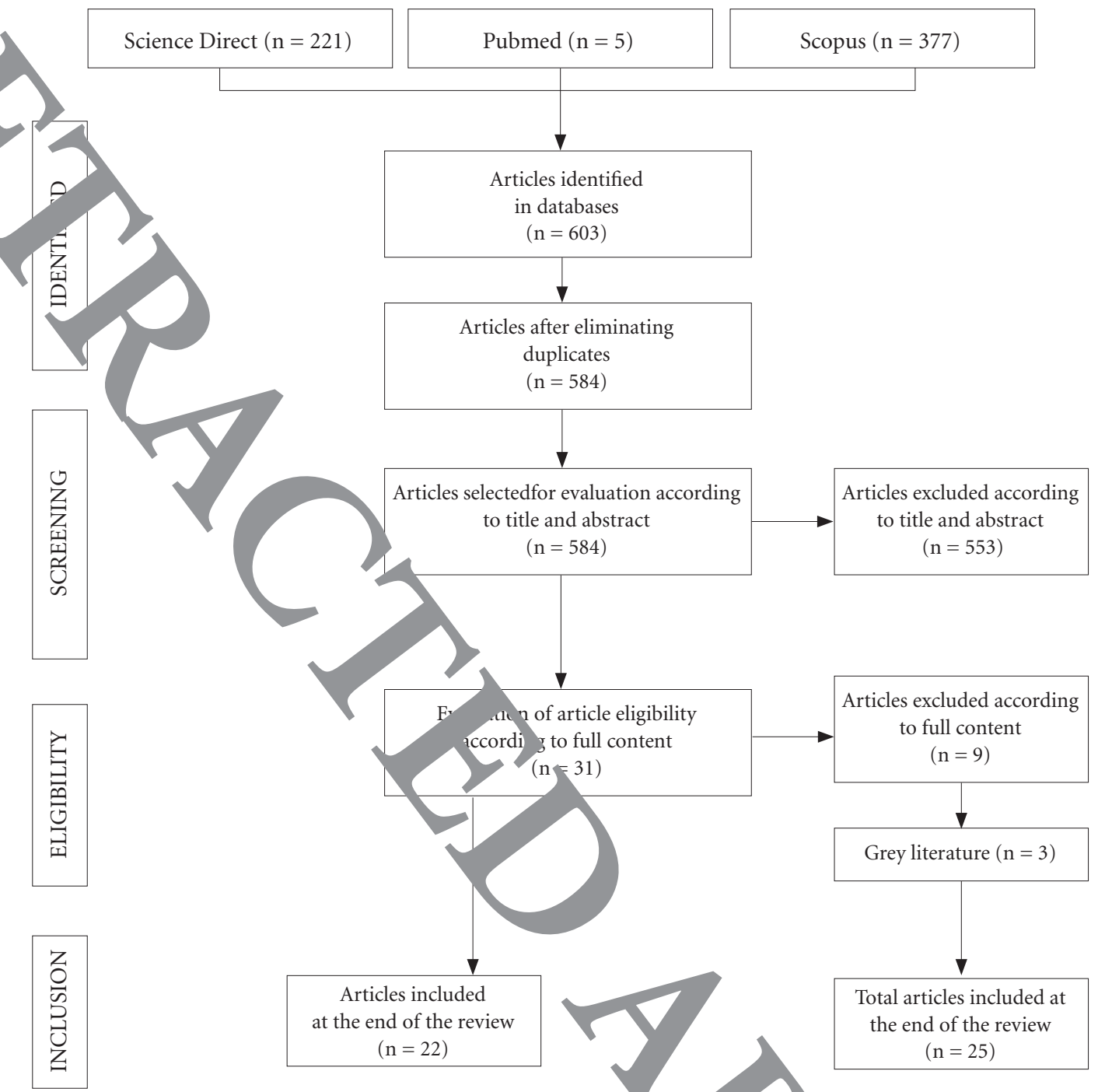

Articles included at the end of the review $(\mathrm{n}=22)$

Figure 1. Search protocol flowchart.

Regarding the year of publication, the year with greatest scientific production around this subject over the period of 11 years was 2013 $(24.32 \%)$. With respect to production trends, production was low $(2.56 \%)$ during the first 4years (2004-2007), but as of 2008, with a production of $10.81 \%$, the number of publications showed an increasing trend until reaching the aforementioned maximum point (Graphic 2).

\section{Food security in rural populations}

Food security is an issue that has not escaped the interest of indigenous and peasant popula- tions. In fact, Briggs \& Mo a ave suggested that food security, together th so i i considered to be "very importar on the ec nomic and nutritional developm ht of the $\mathrm{f}$ ming communities in the Zombw area (Malaw ${ }^{10}$. This report proves that both the and and perception of the population are im at when dealing with food security.

Food insecurity is a predominant atus: the indigenous populations, and Canadiar $\mathrm{Al}^{-}$ munities, particularly those inhabiting the An tic, are no exception ${ }^{3}$. This public health problem caused by multiple determinants, including high levels of poverty, climate change, environmental 


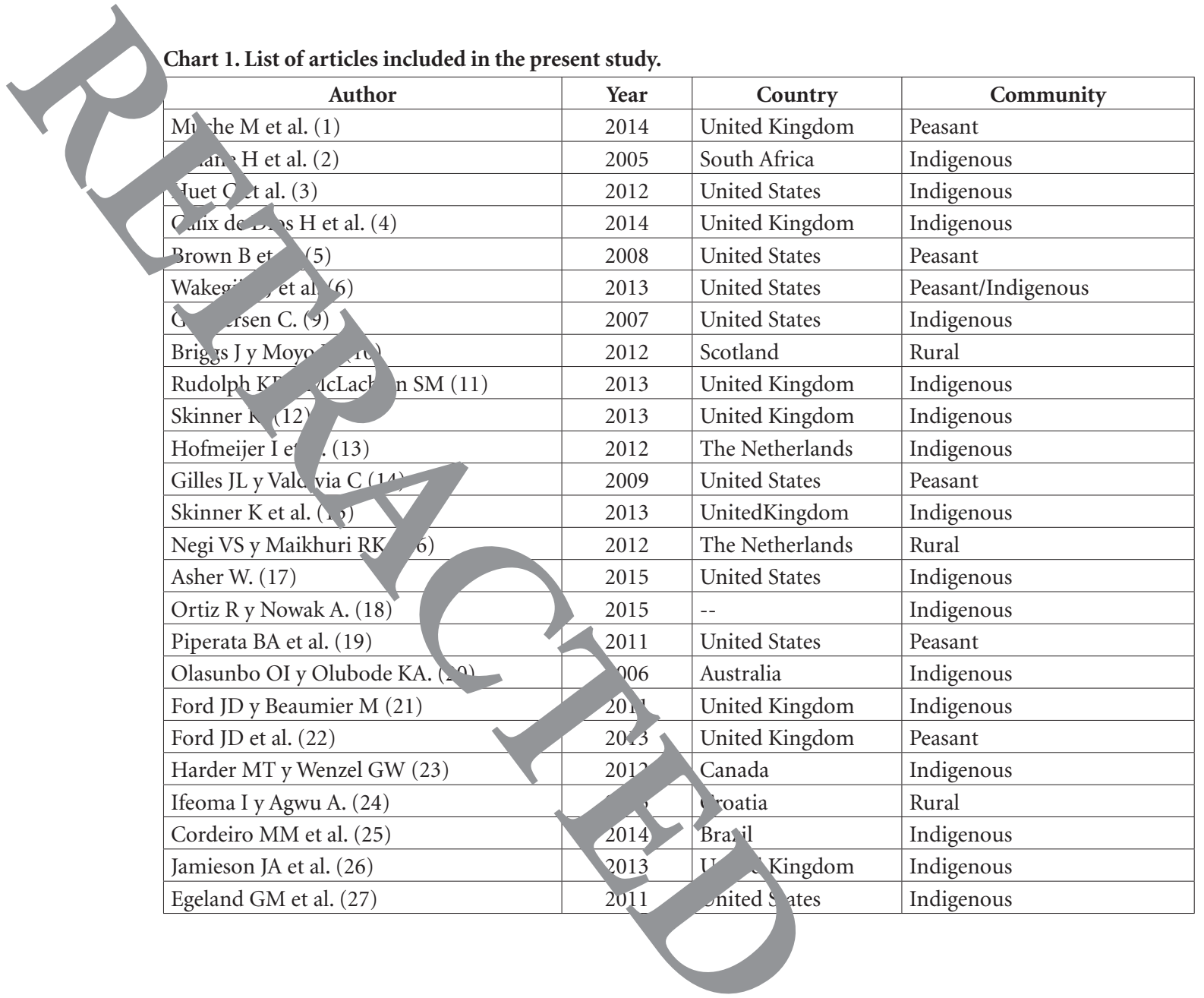

pollution, the introduction of processed foods, the reduction of crop products consumption, and restrictive policies ${ }^{11}$.

For the specific communities of the Arctic, climate change, increases in the prices of the materials needed for hunting, and unemployment have Westernized their culture, and these communities have thus been forced to consume high-cost commercial foods with low nutritional value, leaving aside traditional food and putting food security at risk ${ }^{3}$. This indicates that traditional food systems are increasingly threatened by factors ranging from climate change to the insertion of the nontraditional market; therefore, the affected communities are suffering from long-term food insecurity ${ }^{12}$.

Climate change and its consequences are not exclusively the problem of Arctic communities. For example, the Amazonian population of Peru also suffers from its consequences and the vulnerabilities that the phenomenon generates. Hofmeijer et al. have identified 3 factors arising from climate change that represent health risks to th $\mathrm{c} m$ anities: the generation of food insecurity, $y$ er insecu rity, and the risk of vector-transn ed dis $s$ These communities are particularly ptibl o these circumstances, given their oor mocon itions and inadequate public healtr olicies to co se with $\mathrm{it}^{13}$.

Agricultural sysems are onl sensitive to climate change, and thi oblem sore serious in developing coun les that do $r$ have the necessary tools to minimize the alage caused by the climate-driven deter on of ir agricultural systems. Gilles \& Vardivia ve ident fied a lack of connection between $t$ indigenou producers of the Altiplano and he scientific community that makes climate predi ins in Aymara-speaking communities in Bolivia Peru, in the years 1999-2001, 1999-200 and 2000-2001, respectively. Therefore, despite of forts to provide increasingly accurate weatho forecasts, if there is ineffective communication between the parties, there may be improvements to food security ${ }^{14}$. Thus, biodiversity sustainability is important for some Canadian aboriginal 


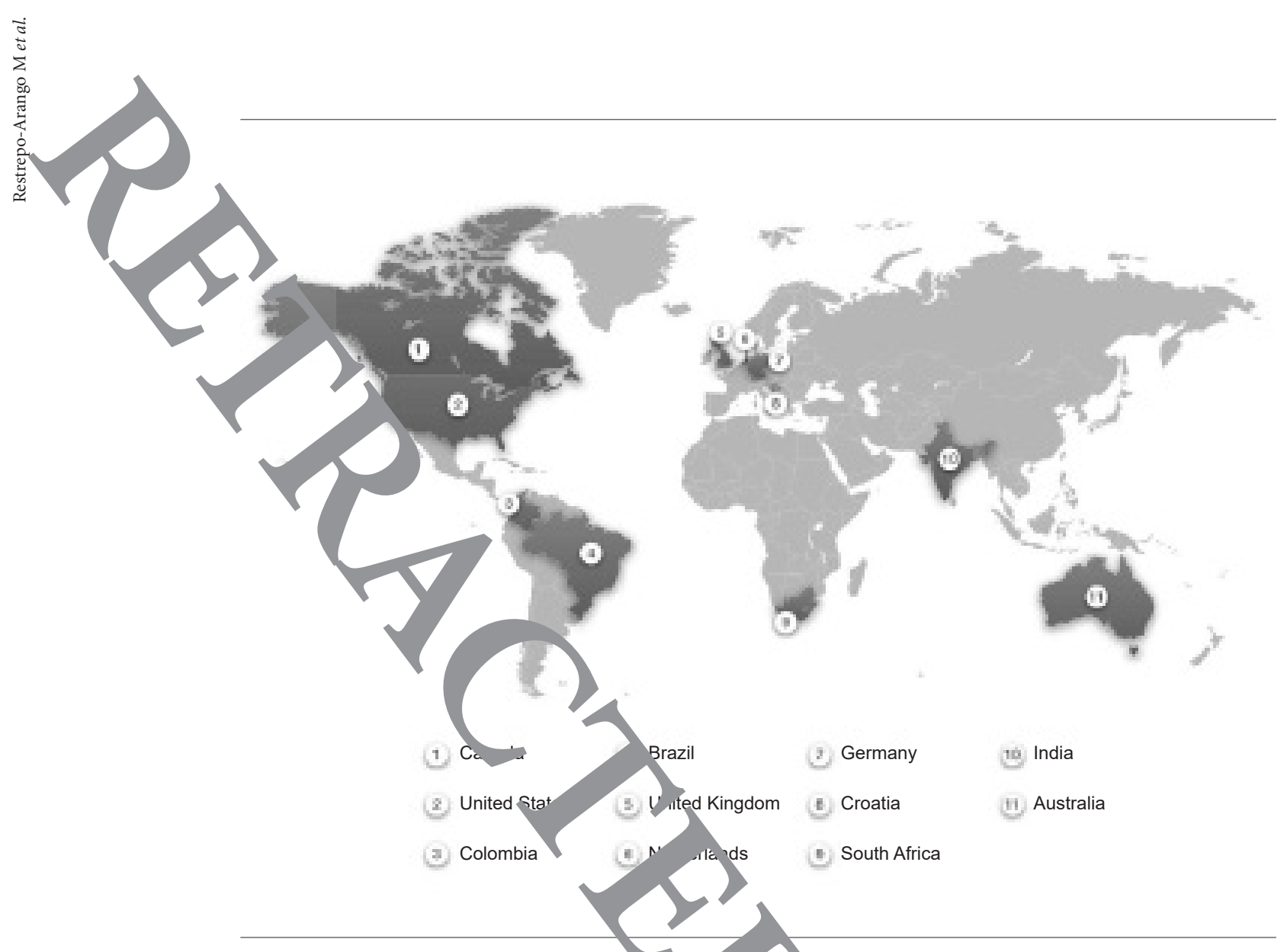

Figura 2. Distribución y localización geogranca d public iones encontradas sobre seguridad alimentaria en poblaciones rurales.

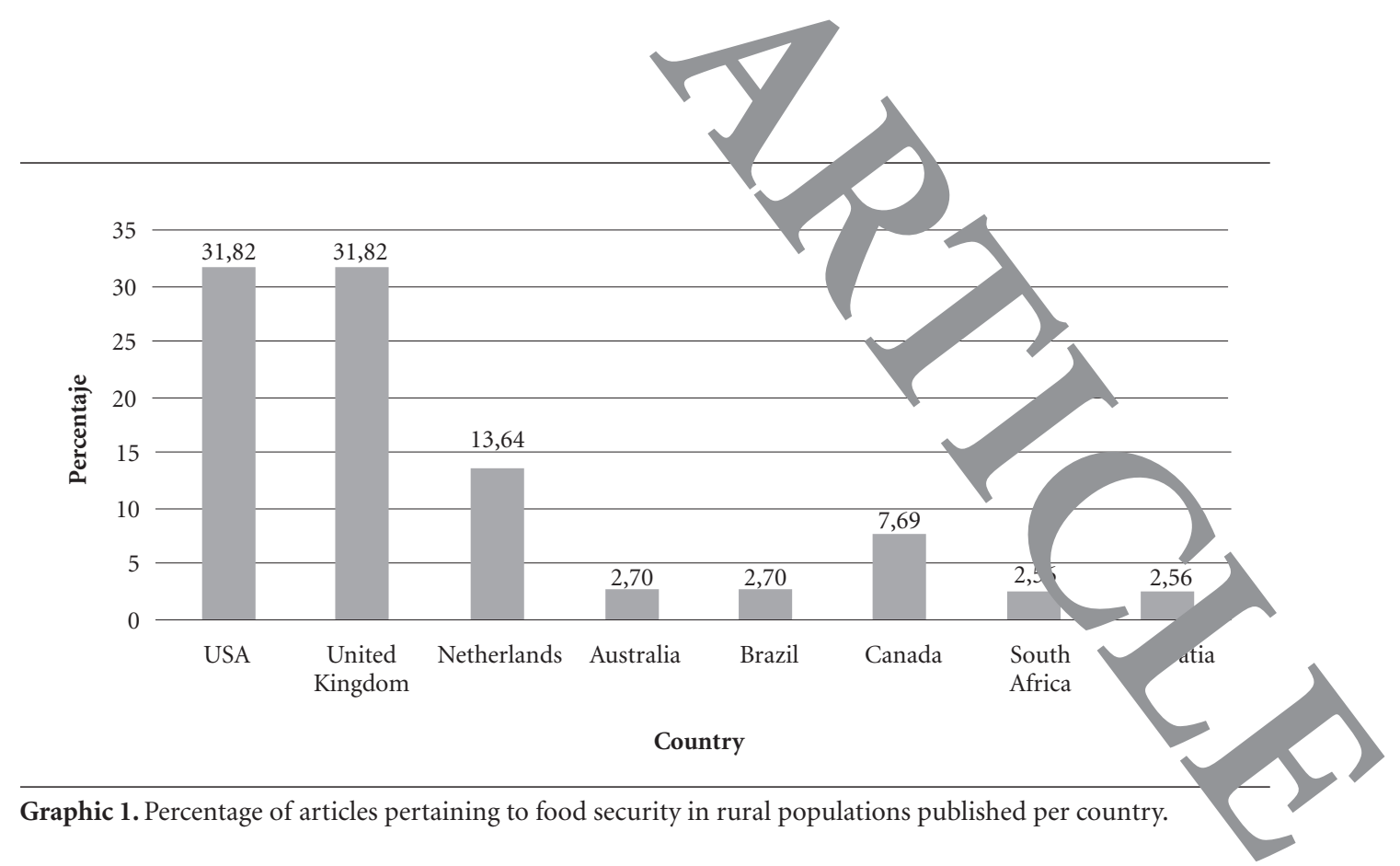




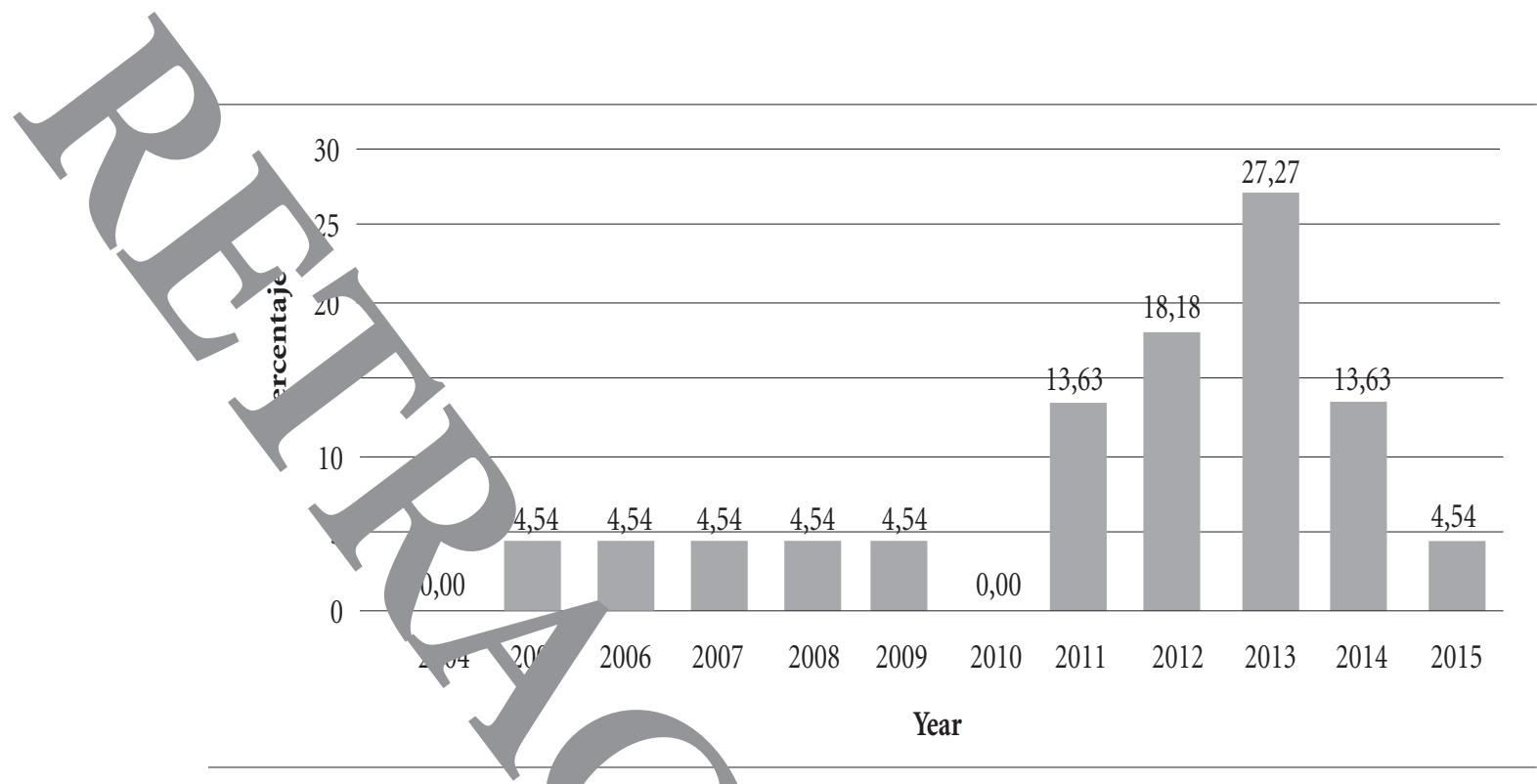

Graphic 2. Percentage of arti es pertaining lo food security in rural populations published per year.

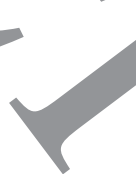

communities, wherein food insecurity problem $^{15}$, and for the indigenous comm nities curity and its determinants of Nepal, livestock biodiversity is of critica $\mathrm{n}$ ral populations portance for sustaining food security and grow ${ }^{\text {th }}$ and for reducing poverty ${ }^{16}$.

In other regions of the world, several studies have indicated that $41 \%$ of the population in Ethiopia lives below the poverty line, with 31.6 million undernourished individuals. In Ethiopia, cases of malnutrition and food insecurity are prevalent in rural areas, with a population of 6 to 7 million people in a chronic state of food insecurity and more than 13 million in variable conditions $^{1}$. Kidane et al. have reported different data, with $50 \%$ of the population in a state of food insecurity and $60 \%$ below the poverty line, on the basis of the daily caloric intake of 2100 per day recommended by the Federal Democratic Republic of Ethiopia ${ }^{2}$.

Low economic status is considered one of the primary predictors of food insecurity, and indigenous communities in the Americas have some of the highest rates of poverty and unemployment. Thus, the scarcity of prevalence data on the status of food insecurity is a cause for concern ${ }^{5}$. An example of the effort being made to face this problem is "The Shoulder-to-Shoulder Global Clinic" located in Santo Domingo, Ecuador, which was established at the University of Kentucky and sought to address the nutritional deficit caused by food insecurity ${ }^{17}$.

Ber se the Amazon is the largest tropical 1 the world, it is the main focus of the world's biodiversity conservation. Amazonian ecosystems are tinuously threatened by human corn nt and climate change, both of which serior affect he food security of the Amazonia populat Thus, economic and social activities a po dlations have always been a matter 1 in and concern. Several studies have dem strated tha market integration has affected the heal ${ }^{1}$ a nutrition of the Amazonian commun, incre sing the incidence of chronic disea es such as weight, obesity, diabetes, and hypertensio

Piperata et al. have cond a a stu-with 469 participants in 2002, ranging age from birth to 77 years; in 2009, 429 inc 1duals participated under the same conditions . Out of the participants evaluated in 2009, 204 h.d olready been evaluated in 2002, meeting the charact tics of a follow-up group. The data were co ected through interviews directed at the househ ld heads, asking them to list all incomes and contri butions to the monthly expenditure. Information on housing and access to basic health services was also collected. Regarding the anthropometric measurements, height, weight, tricipital skin 
fold, upper waist circumference, and body mass index were measured to evaluate nutritional status. The entire process was aimed at determining how communities were being affected by lifestyle anges associated with pathologies, which is al "known as "nutritional transition"19.

Ir rviews, questionnaires, and checklists are of the most common measurement techaques. Sla unbo \& Olubode ${ }^{20}$ have assessed the nutrition Inerability and the status of the Yorul aders Nigeria. In that study, they used a acty erview for non-demographic data and a c stionnaire for demographics; the socioeconon c level wan aked as high, medium, or low, and aith st? was evaluated according to the number of 3 nents. Nutritional vulnerability was evalua wit woun Ged checklist from "The Nutrition Scr ang Initia ve" ${ }^{20}$. Conversely, Brown et al. ${ }^{5}$ ha used a sur,ey with 76 items that collected food s fety, health sta us, and sociodemographic information of co nities between September and Decemb 005 . F i health status, they used the "SF-12 em Health $\mathrm{c}$ tus," which is a validated adaptation of the $6, \mathrm{a} d$ for the measurement of food safe cy sed the "US Household Food Security Su vey Module," which evaluates food safety at homes ioget' these studies demonstrate how 2 differe cthodologies are used to evaluate these aspect.

Another example of the use of intervi was noted in the research conducted by Ford \& Beaumier, who sought to characterize food insecurity in the members of the Inuit community in Igloolik ${ }^{21}$, Nunavut, and to examine the conditions and processes that limited access to food as well as the availability and quality of the food. In that study, 66 semi-structured interviews were conducted at homes, 10 with focus groups comprising community members and the other 19 with local informants and political actors. The focus groups were used to evaluate local strategies and the impact of external stress. According to the information collected, the lack of affordability of food, the low income, the lack of food preferences and knowledge, the low quality and insufficient availability of food products, environmental stress, decreased hunting activity, and elevated harvest costs were factors contributing to food insecurity in this population ${ }^{21}$. However, not all authors agree with the usefulness of predesigned interviews. In the case of "The 18item Core Food Security Module," Gundersen ${ }^{9}$ has argued that the use of this tool to classify the state of food security leads to inaccurate results, given the substantial amount of information that is overlooked; for example, if 2 households are evaluated and one has 8 positive answers and the other has 18 positive answers, both will be categorized under the category of food insecurity with hunger even if there are evident differences in the severity of the situation between the 2 families.

An alternative and rather interesting method has been used by Ford et al. ${ }^{22}$ to assess the use of community food programs in the Canadian Arctic population. The method is called photovoice workshops, where participants are given a camera to photograph their daily life experiences for later evaluation. The participants were asked to present with their photographs the aspects of their day-to-day life that affect their food preferences and the amount of food consumed. This method was effective for the researchers in order to overcome the difficulty of conducting interviews with these communities and also proved to be an interesting experience as the participants themselves were researchers ${ }^{22}$.

Incomes and expenses of families are other useful sources of information for assessing household food security. This aspect was evaluated by Harder \& Wenzel ${ }^{23}$, who examined the low of money and food resources of an extende family at the Inuit community in Clyde RivScotland, to understand the effects of wages and hunting economics on food security. They evaluated employment status, categorized as fulltime t time, temporary, or unemployed, rid he come in dollars, without any specific scale. sets were recorded according to the possess 1 of sno iles, boats, cabins, and truck and hom nersh ${ }^{23}$. While Harder \& Wenzel did not cluc ane their study, in a similar study, Brow! tal. classific income as follows: < 10,000 annu, dollars 10 v the national poverty index, betwee 0,000 ,id 20,000, as close to poverty, and gr ater than $>3 \quad 00$ as a normal poverty level ${ }^{5}$. Ifeoma an $\mathrm{gwu}^{24}$ considered that house size and ac 10 ban ${ }^{1-2}$-dit are determinants of food security $b$ ase any activity that increases income, even ne one oth than agriculture, is a good strates for dealing ith food insecurity ${ }^{24,25}$.

Despite the usefulness of income cssment as an indicator of food security, it is nerally accepted that measuring individual economic as tivities is insufficient for understanding the plete economic situation of the communit ss as a whole because factors, such as sporadic w and household organization, also influence family dynamics ${ }^{23}$. 
s. Weight was evaluated using a solar-powere scale, size a compact stadiometer, and BMI was ac ad ac rding to the $z$-scores given by the arganization ${ }^{25}$. Conversely, Jamieson et ${ }^{6}$ have used bioelectrical impedance analysis o measuro ght and body composition, wher $\mathrm{Lg}$ la et al. ${ }^{27}$ have measured blood biomarkers, sur as 25-hydroxyvitamin D [25(OH)D], compli blor with greater emphasis on hemoglobir valuation nd erythrocyte count, serum ferri $n$, and the er m concentration of saturated fat $\mathrm{s}$, acids and tran -fatty $\operatorname{acids}^{26,27}$.

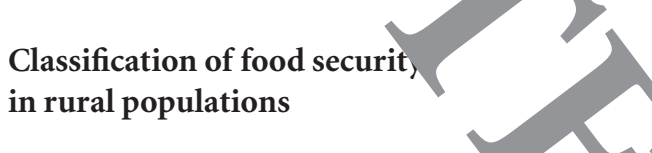

evaluate food security in rural populations; other than their objective, there are no significant similarities among the methods, which hinders the establishment of a consensus method for assessing food security.

\section{Discussion}

Food insecurity in indigenous and peasant populations is a significant public health concern for the scientific community, and over the last 5 years, research in this area has increased. In a period of 11-years evaluated in the present study (2004-2015), there was little scientific activity around this topic, with only $4.54 \%$ of the total publications on this topic; however, for the year 2011, the percentage of publications increased up to $13.63 \%$ in response to the Forum for Food Sovereignty, which had taken place the previous year. Thereafter, scientific production increased encouragingly up to $27.27 \%$ in 2013 , suggesting a surge in research initiatives on this topic. Regarding the amount of production by counLry, although there were some countries, such as

Food security can be classified in dive, se ways depending on the method used to meas it evaluation. Egeland et al. have used the "18-it USDA Food Security Survey Module" survey; interpret the obtained data, they added the following answers: "yes," "often," "sometimes," "almost every month," "some months," and "one or two months." The authors conducted their study according to the food safety classification recommended by Canada's health system as follows: $0-1$ positive response referred to as food security status, $2-5$ positive answers to moderate food insecurity, and greater than or equal to 6 positive answers to severe food insecurity ${ }^{27}$. Another version of this survey, directed atchildren, comprised 10 questions, with categories, such as food security, food insecurity without hunger, and food insecurity with hunger.

An additional scale that has been used for classifying food security in indigenous and peasant populations is the Brazilian scale of food security, which considers households to be at a mild level of food insecurity when the inhabitants have a poor diet or a constant concern of running out of food in little weather at a moderate level when there is a quantitative restriction of food; and at severe levels when there is food deficit or hunger among adults or children in the family ${ }^{25}$. Thus, it is evident how diverse methods, both objective and subjective, can be used to th United Kingdom and the United States with $.82 \%$ of the publications each, the general panorama discouraging, with countries, such as South rica and Croatia, producing only $2.56 \%$ publications each.

The impact of climate change on the food security of the al population is an important facto anc th is expected considering the effect of indy alizatio on the environment and ecosysten . The in munce of climate change lies in the unfar le co itions it generates for hunting as we as $;$ minte tion of migration cycles of species a the vuln ability of agricultural activities In add , he phenomenon also compromises th alth populations and the sustainability o food securi Different studies have demonstrated the $r$ of climate change on the availability, cess, and stability of food products, the re, it now threatens all aspects of food secur in the gen eral population ${ }^{15,16}$.

In terms of agricultural diversit f som communities, the maintenance of a wide $y$ of crops, through numerous small-scale cultivation plots, is the way to achieve food sect ity because variation ensures adaptation to climate change $^{10}$.

The process of globalization has also hindered the achievement of food security in indigenous and peasant populations because of the adoption 
of Westernized diets and lifestyles. In addition, the economic system increasingly depends on salaried jobs, putting aside the traditional acivities of the regions. The introduction of the $V$ zstern market has also led to a greater depende of food stability on purchased foods than on tr tional products. Notably, food security is n ou access to food alone but also about its aality and sufficient nutritional content, which are the re ments that most commercial food prody do no meet. Further, better nutritional alit e correlated with higher market prices. other consequence of globalization is an incr isingly sed iry lifestyle and less balanced die $w h$, $h$ ult in a higher incidence of chronic diseases ach as obesity, hypertension, and diabetes. the which are often regarded as econor and soci l development, negatively affect fo 1 security and the health of populations.

In terms of local, nâtional, and national initiatives addressing the probl of foof insecurity, it can be inferred that su initiatives $r$ ain a scarcely explored topic, with few ativ's from the governments worldwio

tives have been undertaken by coun ries, such as Conclusions Ecuador, Brazil, and the United State nats to combat hunger and food insecurity. that food insecurity is a prevalent status in most of the studies populations and that such a sin number of initiatives has been undertaken in few countries are a matter of concern because they demonstrate a possible lack of funding or interest by governments.

According to the literature reviewed, the measurement, evaluation, and classification techniques applied to the analysis of food security in indigenous and peasant populations widely vary. They range from diverse types of interviews and questionnaires to more interactive methods, such as the use of follow-up photographs. Other authors did not use predesigned formats but created their own methods with the aim to achieve the purpose of the study. Some examples of such models and methods created by organizations include the SF-12 Item Health Status, the U.S. Household Food Security Survey Module, and the 18-item Core Food Security Module. The lack of homogeneity in the measurement methods and the absence of an internationally validated strategy make it difficult to evaluate food security adequately, and concept unification is almost impossible. For example, if food security were to be measured in the indigenous populations of Colombia, there would be no validated method food secu ity. for this group of people, let alone any method created for them, at least in the reports to date. The problem lies in the fact that it is not possible to generate a complete and concise overview of the status of food security of certain populations because there is not a universal instrument that can be applied to evaluate diverse populations. Another limitation is the lack of consensus among authors regarding the variables that should be evaluated to accurately describe the status of food security. Some authors prefer the amount of food, whereas others prefer the type of food or the amount of income and the flow of money. The lack of unification of these concepts is another obstacle faced when attempts are made to generate a single method. Further, there is less information regarding classification; however, it is as divergent as the measurement methods. After examining the different results reported in the reviewed published literature, understanding the basis of the classifications presented by their authors remains challenging owing to the dependency of results on the tool used.

$\mathrm{T}$ maintenance of food security is an increasy challenging task for indigenous and peasant opulations because of various aspects, such as climate change, the Westernization of diets and lifestyles the loss of biodiversity protection $r$ th se opulations. The lack of strategies for copin ith food insecurity reflects the absence of a niversal cospted and validated tool to generate curat overview of the status of

The pop tions studi $d$ in the reviewed research maint ned a ley $\mathrm{m}$ arginality that prevented them from osing b iter food resources and the basic level. of education quired to raise awareness regarding the in ance of self-care, which is one of the leas cussed rents related to nutritional aspects.

The present review demo trated how hethods used to assess food secur y in rural pop $\mathrm{l}_{\mathrm{a}}$ tions differ in basic aspects, such the mes to classify the level of food security ac as the ranges considered to establish variatic is between levels. Regarding this, there are no tonetical references that allow for an adequate inter tion of the measurements performed. This neterogeneity, both in the evaluation methods a d measurements, as well as in their interpretation, demonstrates the importance of research for the 
development of homologous tools and the simultaneous performance of validation processes that allo $\mathrm{v}$ for the recognition of food security in ru. u ulations as an affordable and comparable henor enon, thus leading to the development of polic: and strategies focused on the particularities of he specific communities while also conside the vossibilities of local intervention in sertive and efficient manner.

$1 \mathrm{fact}$, in $\mathrm{m}$ ases, difficulties in accessing these comm a res a result of their remoteness or the ge rap aracteristics of their territories do not $\quad \mathrm{w}$ for the establishment of strategies and foo security nl that are sustainable and that can en ac a ce the minimum food requirements in addit to the development of evaluation and mon ing $r$ these programs, which are the vas or making decisions that affect communities a dimprove tie health conditions.
Therefore, there is a need for food insecurity assessment tools that can be used to provide a basis for the design, evaluation, and monitoring of strategies seeking to promote healthy eating habits, to determine the impact of such strategies. Simultaneously, together with local governments, it is necessary to foster programs that support healthy local food habits from an intercultural perspective, encourage self-care awareness, and promote endogenous processes for the development of public policies appropriate to the actual conditions of these populations.

Finally, the researchers needs to participate more decisively in the development of more affordable food security measurement systems that can be adjusted to the genotypic and cultural characteristics of specific rural populations.
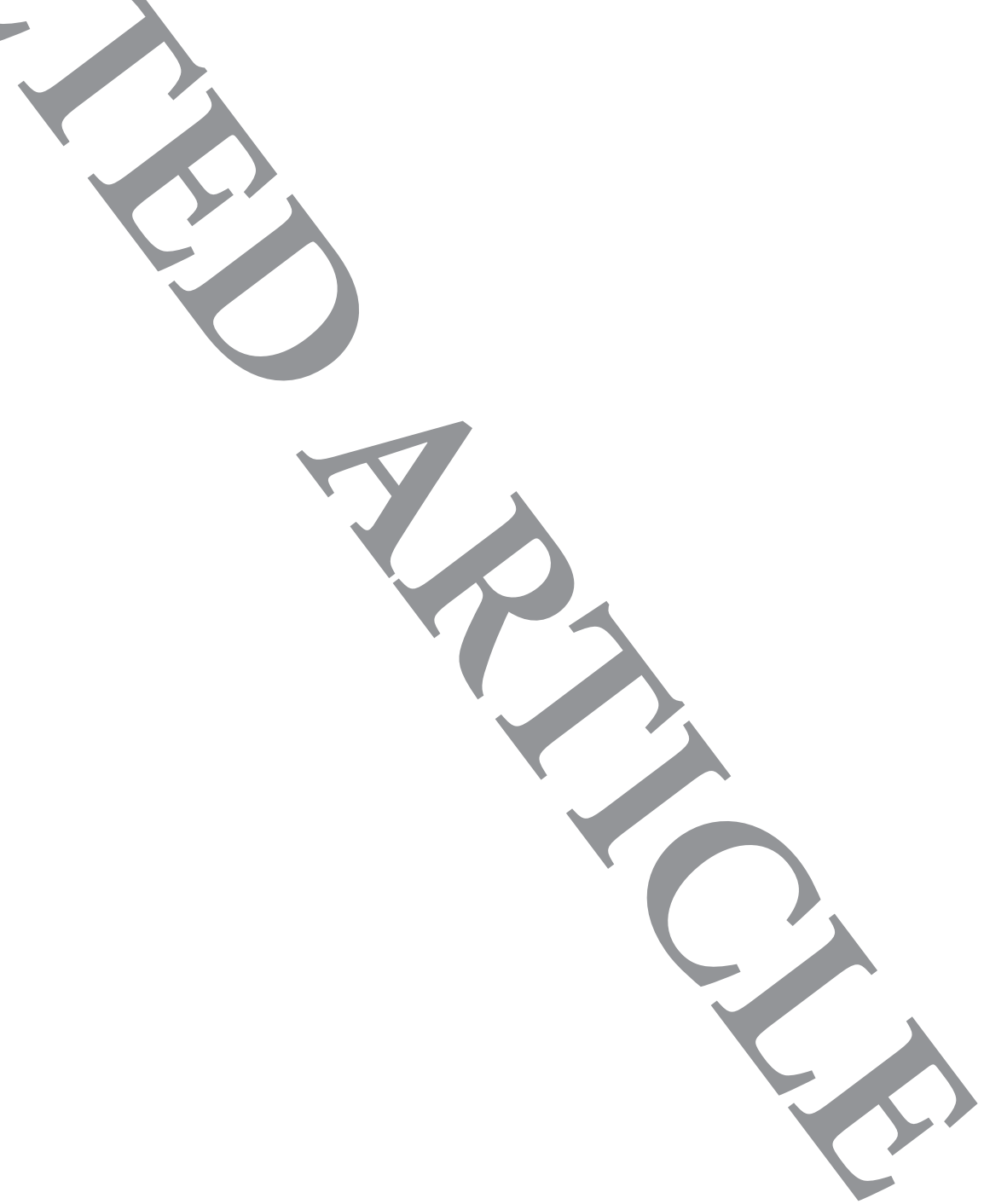


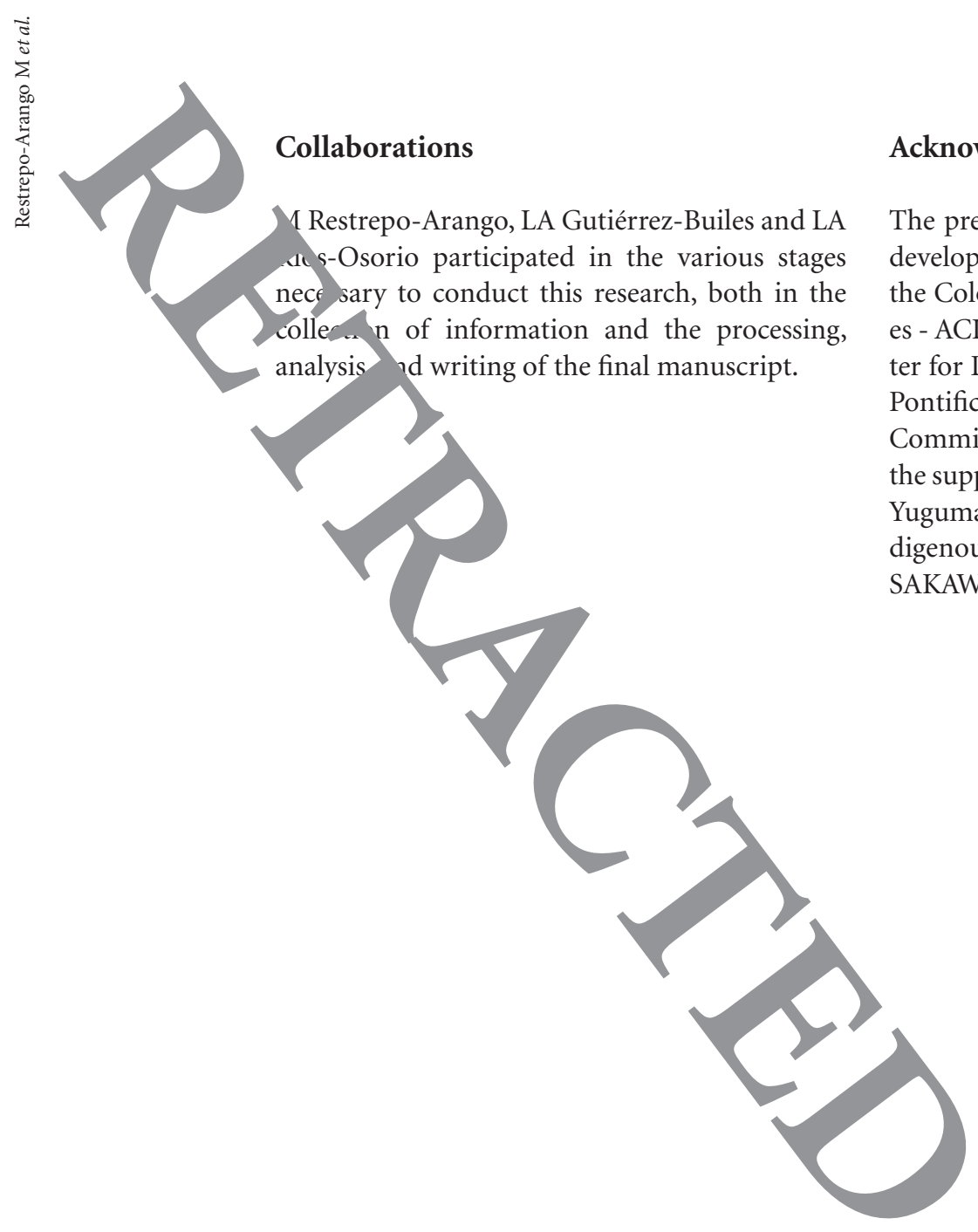




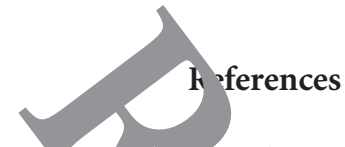

Mit che M, Endalew B, Koricho T. Detrminants of F hold Food Security among Southwest Ethiopia aral h ouseholds. Asian J Agric Res. Academic Journals (c 201, $; 8(5): 248-258$.

2. Ydane $h, A$ mu ZG, Kundhlande G. Causes of household food in urity in koredegaga peasant association, Oromiy a, L hiopia. Agrekon 2005; 44(4):543-560.

3. Hue cosol R geland GM. The prevalence of food in arity is high o the diet quality poor in Inuit communities. J 20 2; 142(3):541-547.

4. Calix de Di Putna H, Alvarado Dzul S, Godek W, Kissm A, Gliessman S. The challenges of measuring $\mathrm{f}$ security and sovereignty in the Yucatán Peninsul Dev Pract. Routl-dge 2014; 24(2):199215.

5. Brown B, Noonan c, Nor Prevalence of Food Insecurity and Health-Ass ted Outcomes and Food Characteristics of North Pla aran weholds. J Hunger Environ Nutr 200.8; 1 .37-53.

6. Wakegijig J, Osborne G, Stat m S, Issaluk D. Collaborating toward improving fo d security in Man vut. Int J Circumpolar Health 2013; 7/ ('unl 1).

7. Santos CMC, Pimenta CAM, Nobre MRC. T strategy for the research question construct and evidence search. Rev Lat Am Enfermagem 20 , 15(3):508511.

8. Urrútia G, Bonfill X. PRISMA declaration: a pron to improve the publication of systematic revic and meta-analyses. Med Clin (Barc) 2010; 135(11):50> 511.

9. Gundersen C. Measuring the extent, depth, and seve. ty of food insecurity: an application to American Indians in the USA. J Popul Econ 2007; 21(1):191-215.

10. Briggs J, Moyo B. The Resilience of Indigenous Knowledge in Small-scale African Agriculture: Key Drivers. Scottish Geogr J 2012; 128(1):64-80.

11. Rudolph KR, McLachlan SM. Seeking Indigenous food sovereignty: origins of and responses to the food crisis in northern Manitoba, Canada. Local Environ 2013; 18(9):1079-1098

12. Skinner K. Prevalence and perceptions of food insecurity and coping strategies in Fort Albany First Nation, Ontario [thesis]. Waterloo: University of Waterloo; 2013.

13. Hofmeijer I, Ford JD, Berrang-Ford L, Zavaleta C, Carcamo C, Llanos E, Carhuaz C, Edge V, Lwasa S, Namanya D. Community vulnerability to the health effects of climate change among indigenous populations in the Peruvian Amazon: a case study from Panaillo and Nuevo Progreso. Mitig Adapt Strateg Glob Chang 2012; 18(7):957-978.

14. Gilles JL, Valdivia C. Local Forecast Communication In The Altiplano. Bull Am Meteorol Soc 2009; 90(1):85-91.

15. Skinner K, Hanning RM, Desjardins E, Tsuji LJS. Giving voice to food insecurity in a remote indigenous community in subarctic Ontario, Canada: traditional ways, ways to cope, ways forward. BMC Public Health 2013; 13(1):427.

16. Negi VS, Maikhuri RK. Socio-Ecological and Religious Perspective of Agrobiodiversity Conservation: Issues, Concern and Priority for Sustainable Agriculture, Central Himalaya. J Agric Environ Ethics 2012; 26(2):491512 .
17. Asher W. Food security factors affect growth in young children in an Ecuadorian Indigenous Community [thesis]. Lexington: University of Kentucky; 2015.

18. Ortiz R, Nowak A, Lavado A, Parker L. Food security in Amazonia. Report for Global Canopy Program and International Center for Tropical Agriculture as part of the Amazonia Security Agenda project, 2013. 2013.p. 1-89. [cited 2015 Jul 5]. Available from: http://globalcanopy. org/sites/default/files/Food security in Amazonia.pdf

19. Piperata BA, Spence JE, Da-Gloria P, Hubbe M. The nutrition transition in amazonia: rapid economic change and its impact on growth and development in Ribeirinhos. Am J Phys Anthropol 2011; 146(1):1-13.

20. Olasunbo OI, Olubode KA. Socio-demographic and nutritional assessment of the elderly Yorubas in Nigeria. Asia Pac J Clin Nutr 2006; 15(1):95-101.

21. Ford JD, Beaumier M. Feeding the family during times of stress: experience and determinants of food insecurity in an Inuit community. Geogr J 2011; 177(1):44-61.

22. Ford JD, Lardeau M-P, Blackett H, Chatwood S, Kurszewski D. Community food program use in Inuvik, Northwest Territories. BMC Public Health 2013; 13(1):970.

23. Harder MT, Wenzel GW. Inuit subsistence, social economy and food security in Clyde River, Nunavut. Arctic 2012; 65(3):305-318.

24. Ceoma I, Agwu A. Assessment of Food Security Situatien among Farming Households in Rural Areas of

\section{K. State, Nigeria. J Cent Eur Agric. 2014; 15(1):94-}

Cordeir MM, Monego ET, Martins KA. Overweight in Goiás' $\mathrm{C}$ ombola students and food insecurity in their fam: Rev Nutr 2014; 27(4):405-412.

26. , w...reson JA, Kuhnlein HV, Weiler HA, Egeland GM. Higher n3-fatty acid status is associated with lower risk of iron depletion ng food insecure Canadian Inuit wom $P$ Health 2013; 13(1):289.

27. Egelanê GM, Inson-Down L, Cao ZR, Sheikh N, Weiler $\mathrm{H} F$ d insecur and nutrition transition combine to fect nu ${ }^{+}$int kes in Canadian arctic communities. SN $\left.^{\prime} 1 ; 14^{1}\right): 1746-1753$.

Article submitted 09/02/2016 Approved 20/07/2016

Final version submitted 22/07/2016 
